SMAD, Rev. Eletrônica Saúde Mental Álcool Drog.

2019 jul.-sept.;15(3):1-9

DOI: 10.11606/issn. 1806-6976.smad.2019.151938

www.revistas.usp.br/smad/

Artículo Original

\title{
Consumo de alcohol entre estudiantes mexicanos de bachillerato
}

\section{Ángel Alberto Puig-Lagunes ${ }^{1}$ \\ (D) https://orcid.org/0000-0003-0177-3921 Ángel Puig Nolasco ${ }^{1}$ \\ (D) https://orcid.org/0000-0003-4876-9727 \\ Luis Enrique Salinas Mendez 1 \\ (D) https://orcid.org/0000-0003-1051-3291 \\ Jesús Enrique Vargas Álvarez ${ }^{1}$ \\ (D) https://orcid.org/0000-0003-4601-4283 \\ Sandra Cristina Pillon² \\ (DD https://orcid.org/0000-0001-8902-7549}

${ }^{1}$ Universidad Veracruzana, campus Minatitlán, Veracruz, México.

2 Universidade de São Paulo, Escola de Enfermagem de Ribeirão Preto, Centro Colaborador de la OPS/OMS para el Desarrollo de la Investigación en Enfermería, Ribeirão Preto, SP, Brasil.
El consumo de alcohol por jóvenes y adolescentes es un fenómeno mundial que ha aumentado en los últimos años. Objetivo: evaluar el patrón de consumo y las percepciones sobre el uso de alcohol por personas cercanas a los estudiantes. Método: se trata de un estudio del tipo transversal y de abordaje cuantitativo, involucrando 940 estudiantes de seis planteles del Colegio de Bachilleres del Estado de Veracruz, México. Resultados: las prevalencias del uso fueron: $57.4 \%$ en la vida, $31.5 \%$ en último año, $25.3 \%$ en el último mes; $7.4 \%$ consumen en nivel problemático del alcohol. Las percepciones negativas sobre el consumo de alcohol fueron evaluadas por padres y madres, predominantemente entre los estudiantes que consumen en bajo riesgo. Conclusión: el consumo de alcohol entre los estudiantes de bachillerato es común, principalmente entre los hombres, con un patrón de inicio es cada vez más precoz, y con la incidencia de intoxicación. Además, estas siempre muy presente en los estudiantes con problemas en las relaciones parentales. Los resultados podrán subsidiar la planificación de programas preventivos en relación al consumo de alcohol en el ámbito escolar.

Descriptores: Consumo de Alcohol, Adolescente, Estudiantes de Bachillerato, Epidemiología, Factores de Riesgo.

\section{Cómo citar este artículo}

Lagunes-Puig AA, Nolasco AP, Mendez LES, Alvarez JEV, Pillon SC. Alcohol consumption in Mexican high school students. SMAD, Rev Eletrônica Saúde Mental Álcool Drog. 2019;15(3):1-9. doi: https://dx.doi.org/10.11606/issn.1806-6976.smad.2019.151938 


\section{Alcohol consumption in Mexican high school students}

Alcohol consumption by young people and adolescents is a worldwide phenomenon that has increased in recent years. Objective: to evaluate the consumption pattern and the perceptions about alcohol use by people close to the Mexican high school students. Method: this is a crosssectional and quantitative approach, involving 940 students from six bachelors courses in the State of Veracruz, Mexico. Outcomes: prevalence of use was $57.4 \%$ in life, $31.5 \%$ in the last year, $25.3 \%$ in the last month; $7.4 \%$ consumed alcohol on a problematic level. Negative perceptions about alcohol consumption were evaluated by fathers and mothers, predominantly among students with low risk consumption. Conclusion: alcohol consumption among baccalaureate students is high, especially among men, with an earlier onset and incidence of intoxication. In addition, it is very present among students with problems in parental relationships. The results may support the development of preventive programs in relation to alcohol consumption in school settings.

Descriptors: Alcohol Consumption, Teens; High School Students, Epidemiology, Risk Factors.

\section{Consumo de álcool entre estudantes mexicanos de bacharelado}

O consumo de álcool por jovens e adolescentes é um fenômeno mundial que tem aumentado nos últimos anos. Objetivo: avaliar o padrão de consumo e as percepções sobre o uso de álcool por pessoas próximas aos estudantes mexicanos de bacharelado. Método: Trata-se de um estudo do tipo transversal e de abordagem quantitativa, envolvendo 940 estudantes de seis cursos de bacharelado do Estado de Veracruz, México. Resultados: As prevalências de uso foram: $57,4 \%$ na vida, $31,5 \%$ no último ano, 25,3\% no último mês; 7,4 \% consumiam alcohol em nível problemático. As percepções negativas sobre o consumo de álcool foram avaliadas por pais e mães, predominantemente entre os estudantes com consumo de baixo risco. Conclusão: 0 consumo de álcool entre estudantes de bacharelado é elevado, principalmente entre os homens, com início cada vez mais precoce e incidência de intoxicação. Além disso, é muito presente entre estudantes com problemas nas relações parentais. Os resultados podem subsidiar o desenvolvimento de programas preventivos em relação ao consumo de álcool no âmbito escolar.

Descritores: Consumo de Álcool, Adolescente, Estudantes de Bacharelado, Epidemiologia, Fatores de Risco. 


\section{Introducción}

El Informe Mundial sobre alcohol y salud estimó que cerca de $5.9 \%$ ( 3.3 millones) de muertes a nivel mundial fueron atribuidas al consumo de alcohol(1). Existe preocupación en relación a los adolescentes; pues constituyen una gran población que lo consume; $40 \%$ de los jóvenes en edad de 15 años o más ha consumido alcohol en los últimos 12 meses; además $16 \%$ de estos bebedores lo consumen excesivamente ${ }^{(1)}$.

El consumo de alcohol por jóvenes y adolescentes es un fenómeno mundial; en México no es la excepción. Una vez que el consumo y el abuso está relacionado entre los principales problemas sociales y de salud pública; en este país resulta con un enorme costo social y de salud por las incapacidades que genera(2) y afecta especialmente a los adolescentes y jóvenes.

Un estudio reporta que una proporción considerada de adolescentes usuarios ocasionales de alcohol podrían continuar consumiendo lo hasta llegar a beber de una manera problemática o tornarse dependiente; una vez que empiezan a ingerir alcohol a temprana edad (13 y 15 años) se pueden potencializar los riesgos en edad adulta(3). Además de que afecta el desempeño académico; al generar faltas y abandono escolar; así como el desarrollo saludable y la transición en la etapa de la adolescencia a la vida adulta. En México el comercio de bebidas alcohólicas a la población menor de 18 años está prohibida; ya que es ilícita en esta población. Sin embargo existe un consumo que es considerado como parte sociocultural en esta población y en esta región ${ }^{(4)}$.

El consumo de alcohol en estudiantes adolescentes ha generado preocupación por parte de las autoridades; educadores; profesionales de salud y familiares sobre las tendencias de consumo en esa población; debido a diversidad de problemas y los conflictos familiares; sociales y de salud resultantes del abuso(5-6). Las Encuestas Nacionales de Adicciones (ENA) realizadas en México(7); muestran incremento significativo en la prevalencia del uso de alcohol en la población de 12 a 17 años; ya que el consumo en la vida pasó de $35.6 \%$ a $42.9 \%$; en el último año de $25.7 \%$ a $30.0 \%$ y en el último mes de $7.1 \%$ a $14.5 \%{ }^{(7)}$. En los estudiantes de 12 años o menos; la prevalencia del consumo de alcohol en la vida fue del $26 \%$; la cual se incrementó a $78.4 \%$ en los estudiantes de 17 años y $83.8 \%$ en los estudiantes mayores de 18 años. Además; en el uso excesivo de alcohol las prevalencias son más elevadas; a medida que aumenta las edades; 12 años o menos (4\%); 17 años $(28.8 \%)$ y entre los estudiantes con 18 años llega a $37.7 \%{ }^{(8)}$. Ese aumento también fue observado tanto en hombres ( $11.5 \%$ a $17.4 \%$ ) como en mujeres $(2.7$ a $11.6 \%)$; especialmente en el consumo del último $\operatorname{mes}^{(7)}$. Estudios realizados en México con estudiantes de bachillerato reportan prevalencias de consumo con grandes variaciones; siendo de 12.2 a $78.8 \%$ en hombres y 7.6 a $66.7 \%$ en mujeres ${ }^{(9-10)}$; y $14.3 \%$ y $66.1 \%$ el consumo en nivel de riesgo; 1.1 y $5 \%$ con síntomas de dependencia y 2.9 y $21.3 \%$ el consumo perjudicial(10-11).

De acuerdo con la Encuesta Nacional de Consumo de Drogas en Estudiantes (ENCODE)(8) la prevalencia del consumo de alcohol en estudiantes de bachillerato destaca el consumo en los hombres; en la vida $56.5 \%$; en el último año $43.8 \%$ y consumo excesivo de alcohol $27.3 \%$; mientras que para las mujeres: $52.1 \% ; 40 \%$ y $21 \%$ respectivamente. En la ciudad de México; el $55.3 \%$ han consumido alcohol en el último mes; sin mostrar diferencias en relación al sexo $(71.4 \%)^{(12)}$. En la Sierra Nororiental del Estado de Puebla; el $83.8 \%$ de los estudiantes consumen alcohol de una a dos veces al mes; siendo la edad promedio de inicio 14.3 años ${ }^{(13)}$.

En el estado de Veracruz; $58.3 \%$ de los adolescentes consumen alcohol; en donde el $40 \%$ consumen en nivel de bajo riesgo; $17.2 \%$ en nivel de riesgo y $1.1 \%$ presentan probable dependencia(11). La prevalencia del consumo de alcohol en las mujeres adolescentes es más elevada; $52 \%$ en la vida y $20 \%$ de éstas lo consume frecuentemente ${ }^{(14)}$. Aunque los hombres consumen más; las mujeres presentan un importante índice de consumo de alcohol; ya que por cada tres hombres adolescentes que presentan probable dependencia hay una mujer; mientras que en la población adulta se presenta $6: 1$ respectivamente. Este panorama es un fenómeno reciente en las mujeres adolescentes; situación que conduce a fortalecer las medidas preventivas y de reducción daños dirigidos a esta población.

La mayoría de los estudios realizados con estudiantes de bachillerato han evaluado el consumo de alcohol de manera aislada(8-11,15-17); por lo cual existe una escasez de datos sobre el uso de alcohol y las percepciones de sus familiares a respecto del consumo por estudiantes mexicanos. Debido al aumento del consumo del alcohol entre adolescentes y la necesidad de emplear estrategias preventivas; de reducción de daños y políticas institucionales direccionadas a esta población. El presente estudio tiene por objetivo evaluar el patrón de consumo y las percepciones sobre el consumo por personas cercanas a los estudiantes.

\section{Método}

Se trató de un estudio de tipo transversal de abordaje cuantitativo. Fue realizado en seis planteles del Colegio de Bachilleres del Estado de Veracruz (COBAEV); en el Sureste del Estado de Veracruz; México.

La obtención de la muestra se realizó mediante el cálculo muestral para proporciones en población finita de 
5145 estudiantes inscritos en el año de 2014; con una confiabilidad del $95 \%$; precisión del $1.5 \%$ y una Za de 0.05 al que le corresponde un valor de $Z$ de 1.96. Para el presente estudio; se incluyeron todos los estudiantes inscritos en segundo; cuarto y sexto semestre de los seis planteles del COBAEV. Fueron seleccionados 940 (100\%) estudiantes; distribuidos de la siguiente manera: 224 (23.8\%) de Cosoleacaque; 191 (20.3\%) Jáltipan; 100 (10.6\%) Chinameca; 109 (11.6\%) Nanchital; $216(23 \%)$ Minatitlán y $100(10.6 \%)$ Zaragoza. Todos los cuestionarios aplicados a los estudiantes fueron respondidos al 100\%; previo de consentimiento informado firmado. Los estudiantes fueron seleccionados de acuerdo a los criterios de inclusión como: estar matriculados en el segundo; cuarto y sexto semestre; estar presente en los salones que se les asignaron para que se les aplicaran los cuestionarios y se tuvo el cuidado de revisar minuciosamente cada cuestionario de manera que no faltara alguna respuesta que era el criterio de exclusión.

Para la recolección de datos; se aplicó tres cuestionarios: (1) Información sociodemográfica conteniendo; edad; sexo; grado; estado civil; religión; ingresos personales y familiares; convivencia con familiares; perdidas emocionales y percepción del uso de las drogas; (2) el Cuestionario de Estudiantes 2006(18) que se basa en la Encuesta Nacional de Adicciones (ENA); contiene 158 preguntas cerradas; divididos en factores de riesgo para el consumo de droga. Sin embargo; para fines de este estudio sólo se tomaron en cuenta 14 ítems relacionados al uso del alcohol y percepciones de ese consumo. Este instrumento ha sido utilizado en diferentes encuestas con adolescentes y escolares; en las cuales se ha probado su validez y confiabilidad en el contexto mexicano(19); y (3) el Test de Identificación de Trastornos Relacionados al Consumo de Alcohol (AUDIT) (20-22). El AUDIT es un instrumento de identificación del uso problemático de alcohol desarrollado por investigadores de la OMS. Se trata de una prueba compuesta por 10 preguntas que evalúa el uso del alcohol en los últimos 12 meses. En este estudio; la clasificación fue considerada: (a) abstemios o bajo riesgo (AUDIT $\leq 7$ ) y el uso de riego o problemático de alcohol (AUDIT $\geq 8$ ) $^{(20-22)}$. Esta prueba presenta validez y confiabilidad y ha sido probada en población mexicana adolescente con una sensibilidad de $90 \%$ y especificidad del $94 \%{ }^{(20-23)}$.

La encuesta se realizó durante el periodo de febrero a julio del 2014. El proyecto fue aprobado por el Comité de Ética e Investigación de la Facultad de Medicina; de la Universidad Veracruzana; campus Minatitlán; Veracruz con el folio CIM-002.

Para el análisis de los resultados se elaboró una base de datos en el programa EPI info versión 6.02. El análisis estadístico se realizó con medidas de tendencia central y dispersión; frecuencias simples y relativas; así como la prueba Chi-cuadrada $\left(C^{2} i^{2}\right)$; el cálculo de razón de momios y sus respectivos intervalos de confianza (IC) al $95 \%$.

\section{Resultados}

Las características sociodemográficas de los estudiantes fueron predominantemente mujeres $(52.8 \%)$; con un promedio de edad de $16.8 \pm 1.0$ años variando entre los 14 y 20 ; solteros (96.9\%); de religión católica (63.6\%) y de bajo ingreso económico familiar mensual (Tabla 1). Entre los estudiantes con nivel problemático del alcohol (AUDIT $\geq 8$ ) fue predominantemente entre $64.2 \%$ de los hombres; de religión católica (64.2\%) y con bajo ingreso familiar $(67.1 \%)$; con valores estadísticamente significativas. Obsérvese que prácticamente doblan las razones de oportunidades entre los hombres (RM 2.1; IC95\% 1.243.66) y tener pérdidas emocionales que aumentan las oportunidades en 1.6 (IC95\% 1.97-2.77) en consumir alcohol en nivel problemático entre los estudiantes con pérdidas emocionales.

En relación a las prevalencias del consumo del alcohol; más de la mitad (57.4\%) consumió en la vida; $31.5 \%$ en último año y $25.3 \%$ en el último mes. La edad promedio del inicio de consumo fue de 12 años; variando entre los 6 y 18 años. En relación con el patrón de consumo de alcohol en los últimos 12 meses; la frecuencia de una o más veces al día fue observada en $45.9 \%$ en los hombres y el patrón de consumo excesivo; en menor frecuencia entre las mujeres (79.6\%) (Tabla 2).

Los tipos de bebidas alcohólicas consumidas por copa fueron: cerveza 390 (41.5\%); destilados 353 (37.6\%); vino 247 (26.3\%); bebidas preparadas (ejemplo: Presidencola; New Mix) 210 (22.3\%); bebidas tipo "cooler"; "viña real" 192 (20.4\%); pulque $71(7.7 \%)$ y alcohol puro o aguardiente 45 $(4.8 \%)$. La frecuencia de embriaguez o la intoxicación alcohólica fue 179 (19\%) en estudiantes que no habían bebido en el último año; 222 (25.6\%) bebieron por lo menos una vez en el último año; 66 (7\%) una vez en el último mes; 53 (5.6\%) dos o tres veces en el último año; $22(2.3 \%)$ una o más veces en la última semana y 398 (42\%) nunca habían bebido alcohol. En cuanto a la frecuencia de los estudiantes de haberse emborrachado; 318 (33.9\%) nunca en el último año; $147(15.6 \%)$ por lo menos una vez en el último año; 36 (3.8\%) una vez en el último mes; 26 (2.8\%) de dos a tres veces en el último mes y 404 (43\%) nunca habían bebido alcohol; con diferencias estadística significancia entre el sexo $(p \leq 0.05)$. 
Tabla 1 - Características sociodemográficas y consumo de alcohol (AUDIT) de los estudiantes de bachillerato de la región Sureste del Estado de Veracruz; México, $2016(n=940)$

\begin{tabular}{|c|c|c|c|c|}
\hline & \multicolumn{2}{|c|}{$\begin{array}{c}\text { Uso problemático } \\
{\left[f^{\star}(\%)\right]}\end{array}$} & \multirow{2}{*}{$\begin{array}{c}\begin{array}{c}\text { Uso de bajo riesgo } \\
{\left[f^{*}(\%)\right]}\end{array} \\
870(92.6)\end{array}$} & \multirow{2}{*}{$\begin{array}{l}\text { Razón de Momios } \\
\text { (IC95\%) }\end{array}$} \\
\hline & TOTAL & $70(7.4)$ & & \\
\hline Grupo de edad (años) & $\begin{array}{l}14-15 \\
16-18 \\
19-20\end{array}$ & $\begin{array}{c}7(10.0) \\
60(85.7) \\
3(4.2)\end{array}$ & $\begin{array}{c}95(10.9) \\
744(85.5) \\
31(3.5)\end{array}$ & $\begin{array}{c}1.00 \\
1.09(0.47-2.7) \\
1.31(0.25-6.15)\end{array}$ \\
\hline Sexo & $\begin{array}{l}\text { Masculino }^{\dagger} \\
\text { Femenino }\end{array}$ & $\begin{array}{l}45(64.2) \\
25(35.7)\end{array}$ & $\begin{array}{l}399(45.8) \\
471(54.1)\end{array}$ & $2.12(1.24-3.66)^{\dagger}$ \\
\hline Estado civil & $\begin{array}{l}\text { Sin pareja } \\
\text { Con pareja }\end{array}$ & $\begin{array}{c}68(97.1) \\
2(2.8)\end{array}$ & $\begin{array}{c}835(95.9) \\
35(4.0)\end{array}$ & $0.7(0.11-3.11)$ \\
\hline Religion & $\begin{array}{l}\text { Nenhuma } \\
\text { Católica }^{\dagger} \\
\text { Otras }\end{array}$ & $\begin{array}{c}9(12.8) \\
49(64.2) \\
12(17.1)\end{array}$ & $\begin{array}{l}130(14.9) \\
549(63.5) \\
191(21.9)\end{array}$ & $\begin{array}{c}0.85(0.38-1.86) \\
1.00 \\
-\end{array}$ \\
\hline Ingresso Familiar & $\begin{array}{c}\$<1999 \\
\$ 2000->\$ 5000^{\ddagger}\end{array}$ & $\begin{array}{l}29(67.1) \\
41(24.6)\end{array}$ & $\begin{array}{l}455(80.0) \\
415(14.6)\end{array}$ & $0.65(0.38-1.09)$ \\
\hline Convivencia & $\begin{array}{c}\text { Padres } \\
\text { Familiares } \\
\text { Esposa/Pareja }\end{array}$ & $\begin{array}{c}58(82.8) \\
12(17.1) \\
-(-)\end{array}$ & $\begin{array}{c}756(86.8) \\
104(11.9) \\
10(1.04)\end{array}$ & $\begin{array}{c}1.00 \\
1.37(0.68-2.73)\end{array}$ \\
\hline Perdidas emocionales & $\begin{array}{l}\mathrm{Si} \\
\text { No }\end{array}$ & $\begin{array}{l}32(45.7) \\
38(54.2)\end{array}$ & $\begin{array}{l}395(45.4) \\
575(66.0)\end{array}$ & $1.64(1.97-2.77)^{\dagger}$ \\
\hline Percepción del efecto de las drogas & $\begin{array}{l}\text { Positiva } \\
\text { Negativa }\end{array}$ & $\begin{array}{l}10(14.2) \\
60(85.7)\end{array}$ & $\begin{array}{l}104(11.9) \\
766(88.0)\end{array}$ & $1.23(0.57-2.59)$ \\
\hline
\end{tabular}

$*_{\mathrm{f}}=$ frecuencia; ${ }^{+}$Valor de $p \leq 0.05(\mathrm{n}=940)$

Tabla 2 - Patrón de consumo de alcohol (AUDIT) en los estudiantes de bachillerato de la región Sureste del Estado de Veracruz; México $2016(n=940)$

\begin{tabular}{|c|c|c|c|}
\hline & & $\begin{array}{c}\text { Hombrens }\left[\mathbf{f}^{*}(\%)\right] \\
444(47.2)\end{array}$ & $\begin{array}{c}\text { Mujeres }\left[f^{\star}(\%)\right] \\
496(52.8)\end{array}$ \\
\hline \multirow{4}{*}{ Frecuencia de consumo } & Nunca & $201(45.2)$ & $27(56.0)$ \\
\hline & Raramente & 204 (45.9) & $187(37.7)$ \\
\hline & Ocasionalmente $^{\dagger}$ & $28(6.3)$ & $24(4.8)$ \\
\hline & Frecuentemente & $11(2.4)$ & $7(1.4)$ \\
\hline \multirow{6}{*}{$\begin{array}{l}\text { Número de dosis consumida } \\
\text { en un día normal }\end{array}$} & Nunca & $125(28,1)$ & $152(30,6)$ \\
\hline & $1-2$ & $224(50,4)$ & $233(46,9)$ \\
\hline & $3-4$ & $35(7,8)$ & $34(6,8)$ \\
\hline & $5-6$ & $19(4,3)$ & $12(2,4)$ \\
\hline & $7-9$ & $7(1,6)$ & $8(1,6)$ \\
\hline & 10 ou más & $34(7,6)$ & $57(11,4)$ \\
\hline \multirow{5}{*}{$\begin{array}{l}\text { Frecuencia de uso de } 6 \text { o más } \\
\text { dosis en una sola ocasión } \\
\text { (embriaguez) }\end{array}$} & Nunca & $26(5,8)$ & $35(7,0)$ \\
\hline & Menos de una vez por mes & $330(74,3)$ & $395(79,6)$ \\
\hline & Mensual $^{\dagger}$ & $69(15,5)$ & $53(10,6)$ \\
\hline & Semanal & $13(2,9)$ & $13(2,6)$ \\
\hline & Diario o case diario & $6(1,3)$ & $-(-)$ \\
\hline
\end{tabular}

$\mathrm{f}^{*}=$ frecuencia; ${ }^{\dagger}$ Valor de $p \leq 0.05,(\mathrm{n}=940)$

De acuerdo con los estudiantes; las percepciones del consumo de alcohol es evaluado por el padre; la madre; los maestros y los amigos; en donde todos lo ven mal; con predominancia entre los que consumen en bajo riesgo $(p<0.05)$. La percepción del peligro respecto al consumo de alcohol fue referida como peligroso y muy peligroso por la mayoría de las personas al entorno de los estudiantes con consumo de bajo riesgo. Se observan elevados porcentajes de la madre y el padre en la percepción negativa del uso de alcohol. Entre los estudiantes; $92.6 \%$ consumían el alcohol en nivel de bajo riesgo o eran abstinentes y $7.4 \%$ en el uso problemático (AUDIT) (Tabla 3).

La presencia de problemas familiares debido al consumo del alcohol se presentó en $37.2 \%$ de los estudiantes; siendo $12.4 \%$ con el padre; $1.2 \%$ con la madre; $3.5 \%$ con el hermano (a); y $19.9 \%$ otros parientes cercanos. Con respecto a peleas en la familia; dos de cada diez estudiantes manifestaron que es común que se peleen sus padres. Cuando se les pregunta si esta pelea estuvo asociada al consumo de alcohol; $15.5 \%$ respondieron positivamente. 
Tabla 3 - Percepción del consumo de alcohol en los estudiantes de bachillerato de planteles del COBAEV de la región Sureste del Estado de Veracruz, México 2016, $(n=940)$

\begin{tabular}{|c|c|c|c|}
\hline & Percepciones & $\begin{array}{c}\text { Uso problemático } \\
{\left[f^{\star}(\%)\right]}\end{array}$ & $\begin{array}{c}\text { Uso de bajo riesgo } \\
{\left[f^{*}(\%)\right]}\end{array}$ \\
\hline & & $70(7.4)$ & $870(92.5)$ \\
\hline Madre & $\begin{array}{c}\text { Bien } \\
\text { Ni bien, ni mal } \\
\text { Mal }^{+}\end{array}$ & $\begin{array}{c}1(1.4) \\
10(14.3) \\
59(84.3)\end{array}$ & $\begin{array}{c}4(0.45) \\
57(6.5) \\
809(92.9)\end{array}$ \\
\hline Padre & $\begin{array}{c}\text { Bien } \\
\text { Ni bien, ni mal } \\
\text { Mal }^{+}\end{array}$ & $\begin{array}{c}2(2.8) \\
14(20.0) \\
54(77.1)\end{array}$ & $\begin{array}{c}5(0.57) \\
83(9.5) \\
782(89.8)\end{array}$ \\
\hline Maestro (a) & $\begin{array}{c}\text { Bien } \\
\text { Ni bien, ni mal } \\
\text { Mal }^{\dagger}\end{array}$ & $\begin{array}{c}1(1.4) \\
14(20.0) \\
55(78.6)\end{array}$ & $\begin{array}{c}10(1.1) \\
85(9.7) \\
775(89.0)\end{array}$ \\
\hline Amigo (a) & $\begin{array}{c}\text { Bem } \\
\text { Nem bem nem mal } \\
\mathrm{Mal}^{\dagger}\end{array}$ & $\begin{array}{l}10(14.3) \\
29(41,4) \\
51(72,8)\end{array}$ & $\begin{array}{c}28(3.2) \\
195(22.4) \\
647(74.4)\end{array}$ \\
\hline $\begin{array}{l}\text { Consumir } \\
\text { alcohol }\end{array}$ & $\begin{array}{l}\text { No es peligroso } \\
\text { Es peligroso } \\
\text { Es muy peligroso*† }\end{array}$ & $\begin{array}{c}6(8.6) \\
47(67.1) \\
17(24.3)\end{array}$ & $\begin{array}{c}30(3.4) \\
312(35.9) \\
528(60.7)\end{array}$ \\
\hline
\end{tabular}

$*_{\mathrm{f}}=$ frecuencia; ${ }^{+}$Valor de $p \leq 0.05(\mathrm{n}=940)$

\section{Discusión}

El presente estudio evaluó el uso y las percepciones sobre el alcohol en una muestra de estudiantes; compuesta en su mayoría por mujeres; solteras; católicas; con bajo ingreso económico familiar. Estos datos probablemente reflejen la localización geográfica en que la investigación fue realizada. Datos similares fueran reportados en estudios realizados con estudiantes de bachillerato de diferentes partes del país ${ }^{(4,9-10,13)}$.

El consumo de alcohol ha sido cada vez un comportamiento común entre los estudiantes y la prevalencia del consumo es elevada $(57.4 \%$ en la vida; $31.5 \%$ en último año y $25.3 \%$ en el último mes); datos que se corroboran con los reportes en otros bachilleratos del país(8-13); aunque se encuentran por debajo de acuerdo a otros reportes ${ }^{(12)}$. La edad promedio de inicio de consumo de alcohol en este estudio fue menor en comparación con la literatura(13-14) que se encontraron una edad promedio de 14.3 años; lo que indica un consumo del alcohol más temprano.

La frecuencia de consumo de alcohol de una o más veces al día en los últimos 12 meses fue de $45.9 \%$ para los hombres; resultados similares a otros estudios ${ }^{(9-12)}$; sin embargo; la ENCODE $2014^{(8)}$ reporta prevalencias superiores. En conjunto estos datos indican que sigue prevaleciendo mayor consumo de bebidas alcohólicas en los hombres comparado con las mujeres ${ }^{(9-12)}$. Además observamos índices crecientes del consumo en las mujeres; comparados a los de la literatura(9,14-15).

El consumo en el patrón binge (embriaguez) fue predominante en los hombres; valores superiores al reportado por Villatoro Velásquez et al.(12). La bebida de preferencia por estudiantes corrobora al de la literatura nacional( ${ }^{(7-8,13,24)}$. Quizá el consumo de la cerveza entre estudiantes de bachillerato como ya ha sido reportado en otros estudios en México(25) se deba a diversos factores como; bajo costo; accesibilidad para conseguirla; permisividad familiar; aunada al clima cálido-húmedo del Sur del Estado de Veracruz.

El consumo en el patrón binge en los estudiantes fue de $7 \%$ una vez en el último mes; cifra que se encuentra por debajo de la media nacional $(14.5 \%)^{(8)}$ y en estudiantes de bachillerato $(9.9 \%)^{(25)}$; un hecho que quizá pueda deberse al bajo ingreso económico personal y familiar; así como la alta frecuencia de católicos; lo anterior ha sido evaluado; en donde encontraron que los jóvenes sin religión o practicantes de otras religiones tienen una mayor prevalencia del abuso del alcohol(19,24).

Sobre la percepción de riesgo a respecto del consumo; los adolescentes refirieron que es mal visto por sus padres; maestros y amigos; datos que corrobora a los dados de la ENCODE(8). Por otra parte; mitad de los estudiantes percibieron que el consumo de alcohol es peligroso y $43.7 \%$ lo ve como muy peligroso; coincidiendo con la media nacional( ${ }^{(8)}$ y estudios de la $\mathrm{Cd}$. de México ${ }^{(11 ; 24)}$ en donde además reportan un aumento en la percepción de peligroso (78.4\%) en las personas que no lo consumen. Si bien es cierto que en México la prevalencia del consumo de alcohol es elevada; éste se concibe como parte integral de la influencia social de la cultura; los amigos; la familia; redes sociales y los medios de comunicación masiva. Además; se ha encontrado que las principales razones del consumo de alcohol en la adolescencia se deban a la curiosidad; por llamar la atención; por formar parte de un grupo de amigos; por liderazgo en un grupo; por imitación; resolver problemas en su vida y por gusto(25). Por lo consiguiente es necesario hacer más énfasis en todos los niveles educativos; social y 
familiar acerca de los riesgos reales que provoca el consumo de bebidas alcohólicas.

En relación a los niveles de riesgo (AUDIT); de los estudiantes $92.2 \%$ eran abstemios o presentaban bajo riesgo y $7.8 \%$ uso de riesgo o abuso; resultados que difieren a otros estudios en que reporta un uso de mayor riesgo o abuso (810,12-14,21). Esto en parte; puede ser debido a las condiciones socioeconómicas y religiosas; aunado a que varios de los planteles están ubicados en cabeceras municipales semiurbanizadas y algunas en zonas indígenas; que aún tiene arraigadas sus costumbres ancestrales; además de que no existen tantos antros de vicio que pudieran fomentar el consumo del alcohol.

\section{Conclusión}

El consumo de alcohol entre los estudiantes de los distintos planteles del COBAEV es un comportamiento común principalmente entre los hombres; aunque hay que destacar la presencia de un continuo incremento del uso entre las mujeres. Un factor importante y que es motivo de atención para la prevención y reducción en el consumo de alcohol en los adolescentes; es que la edad promedio de inicio es cada vez más precoz; además de la existencia intoxicación; abuso en el consumo de alcohol a tan corta edad en una parte de la población; a pesar de considerarlo como peligroso y que las personas cercanas tenían una mala percepción de su consumo.

El consumo de alcohol estuvo siempre muy presente en los problemas familiares; principalmente por los padres. Los resultados podrán contribuir para la planificación de programas y acciones preventivas en relación al consumo de bebidas alcohólicas en el ámbito escolar; involucrando a maestros; padres de familia y estudiantes.

\section{Referencias}

1. World Health Organization. Global status report on alcohol and health. [Internet]. Geneva (SW): WHO. 2014. [cited 201820 Nov]. Available from: http://apps.who.int/ iris/bitstream/handle/10665/112736/9789240692763_ eng. pdf? sequence $=1$ \&isAllowed $=y$

2. García Rivas ML, Barriguete Mázmela B. Actualidades en adicciones; consumo de alcohol y tabaco en México. México; D.F. Ediciones Berit FGV; 2012. p. 293.

3. Hernández López T, Roldán Fernández J, Jiménez Frutos A, Mora Rodríguez C, Sánchez-Garnica DE, Pérez
Álvarez MT. The starting age of drugs consumption as an indicator for problematic consumption. Psychosoc Intervention. [Internet]. $2009 \mathrm{Dec}$ [cited $2018 \mathrm{Nov}$ 20]; 18(3):199-212. Available from: http://scielo. isciii.es/scielo.php?script=sci_arttext\&pid=S113205592009000300002\&lng=es.

4. Díaz MLR, Díaz MA, Hernández ACA, Fernández VH, Solís TC, Narro RJ. Hazardous and harmful drinking among Mexican adolescents: prevalence and predictive factors. Salud Mental. [Internet]. 2009 Dic [cited 2018 Nov 20]; 32(6):447-58. Available from: http://www. scielo.org.mx/scielo.php?script=sci_arttext\&pid=S0185$33252009000600002 \& \operatorname{lng}=$ es.

5. Puig-Nolasco A, Cortaza-Ramirez L, Pillon SC. Alcohol use among Mexican medical students. Rev. Latino-Am. Enfermagem [Internet]. 2011 June [cited 2018 Nov 20]; 19(spe):714-21. Available from: http://www. scielo.br/scielo.php?script=sci_arttext\&pid=S0104$11692011000700008 \&$ Ing=en. http://dx.doi. org/10.1590/S0104-11692011000700008.

6. Pantoja PJ. Expectativa y su relación con el nivel de consumo de alcohol en una población de bachillerato. Universidades. [Internet]. 2010 [cited 2018 Nov 20]; (46):35-64. Available from: http://www.redalyc.org/ articulo. 0 a id $=37318636005$

7. Medina-Mora ME, Villatoro-Velázquez JA, Fleiz-Bautista C, Téllez-Rojo MM, Mendoza-Alvarado LR, Romero Martínez M., et al. Encuesta Nacional de Adicciones 2011: Reporte de Alcohol. [Internet] México D.F: Instituto Nacional de Psiquiatría Ramón de la Fuente Muñiz; Instituto Nacional de Salud Pública.; Secretaría de Salud; 2012. [Acceso 20 nov 2018]. Disponible en: http://www. conadic.salud.gob.mx/pdfs/ENA_2011_ALCOHOL.pdf

8. Villatoro-Velázquez JA, Fregoso Ito D, Bustos Gamiño M, Oliva Robles N, Mujica Salazar A, Martín del Campo Sánchez R. Encuesta Nacional de consumo de drogas en estudiantes (ENA); 2014: Reporte de Alcohol. México DF; (Méx): Instituto Nacional de Psiquiatría Ramón de la Fuente Muñiz. Comisión Nacional Contra las Adicciones; Secretaría de Salud; 2015. [Acceso 20 nov 2018]. Disponible en:http://www.conadic.salud.gob.mx/pdfs/ investigacion/ENCODE_DROGAS_2014.pdf

9. Tamez-Rodríguez VA, Cerda-Flores RM, Marín-Peña E, Machado-Cervantes JM, Dávila-Canales R, Rodríguez Aguilar L. et al. Evaluación del consumo de alcohol y sus motivaciones en estudiantes del noreste de México. Medicina Universitaria. [Internet]. 2013 [Acceso 20 nov 2018];15(58):3-9. Disponible en: http://www. elsevier.es/en-revista-medicina-universitaria-304pdf-X1665579613949956 
10. Rivas-Acuña V, Carrillo-Hernández L, Hernández -Díaz V, Hernández Carrillo FC. Consumption of alcohol in students of an educational centerof average top level. Villahermosa Tabasco; Mexico. Rev Enferm Herediana. [Internet]. 2011 Dic [Acceso 20 nov 2018]; 4(2):71-6. Disponible en: https://faenf.cayetano.edu.pe/images/ pdf/Revistas/2011/febrero/05\%20ALCOHOL.pdf

11. Hernández-Cortaza BA, Cortez-Ramírez L, Costa ML Junior. Consumption of alcohol and depression in students of a public school of Coatzacoalcos. Veracruz; México. SMAD Rev Eletrônica Saúde Mental Álcool Drogas. [Internet]. 2012 Dec [cited 2018 Nov 20]; 8(3):142-7. Available from: http://pepsic.bvsalud. org/scielo.php?script=sci_arttext\&pid=S180669762012000300006\&lng=pt\&tlng=.

12. Villatoro Velázquez JÁ, Gaytán Flores F, Moreno López M, Gutiérrez López ML, Oliva Robles N, Bretón Cirett $M$, et al. Tendencias del uso de drogas en la Ciudad de México: Encuesta de Estudiantes del 2009. Salud Mental. [Internet]. 2011 Abr [Acceso 20 Nov 2018]; 34(2):81-94. Disponible en: http://www.scielo. org. $\mathrm{mx} /$ scielo.php?script $=$ sci_arttext\&pid=S018533252011000200001\&lng=es.

13. Bello-Rebolledo G, Rosas-Martínez U, BáezHernández FJ. Adolescent Alcohol Consumption in Northeastern Sierra of Puebla. Desarrollo Cient Enferm. [Internet]. 2011 [Acceso 20 Nov 2018]; 19(10):329-33. Disponible en: http://www.index-f.com/ dce/19pdf/19-329.pdf

14. Cortaza Ramírez L, Villar Luis MA, Puig Nolasco

A. Consumo de alcohol entre estudiantes Mexicanos de medicina. Rev. Latino-Am. Enfermagem. [Internet]. Junho 2011 [Acesso 20 nov 2018]; 19(spe): 714-21. Disponível em: http://www. scielo.br/scielo.php?script $=$ sci_arttext\&pid $=$ S0104$11692011000700008 \&$ Ing=en. http://dx.doi. org/10.1590/S0104-11692011000700008.

15. González-González A, Juárez García F, Solís Torres C, González-Forteza C, Jiménez Tapia A, Medina-Mora ME, Fernández-Varela Mejía H. Depresión y consumo de alcohol y tabaco en estudiantes de bachillerato y licenciatura. Salud Mental. [Internet]. 2012 [Acesso 20 nov 2018]; 35(1):51-5. Disponible en: http://www. redalyc.org/articulo.oa?id $=58223290008$

16. Benjet C, Borges G, Méndez E, Casanova L, MedinaMora ME. Adolescent alcohol use and alcohol use disorders in Mexico City. Drug Alcohol Dependence. [Internet]. 2014 [cited 2018 Nov 20]; (136):43-50. DOI: $10.1016 /$ j.drugalcdep.2013.12.006

17. Telumbre-Terrero JY, Sánchez-Jaimes BE. Consumo de alcohol en adolescentes del Estado de Guerrero;
México. Salud y Drogas. [Internet]. 2015. [Acesso 20 nov 2018];15(1):79-86. Disponible en: http://www. redalyc. org/articulo. 0 a id $=83938758008$

18. Villatoro J, Oliva N, Mujica R, Fregoso D, et al. Panorama actual del consumo de sustancias en estudiantes de la Ciudad de México. Ciudad de México: Instituto Nacional de Psiquiatría Ramón de la Fuente Muñiz. Administración Federal de Servicios Educativos en el Distrito Federal. Instituto para la atención y prevención de las adicciones; 2015. doi: 10.17711/ SM.0185-3325.2016.023

9. González-Forteza C, Solís-Torres C, Jiménez-Tapia A, Hernández-Fernández I, González-González A, et al. Confiabilidad y validez de la escala de depresión CES-D en un censo de estudiantes de nivel medio superior y superior; en la Ciudad de México. Salud Ment. [Internet]. 2011 Feb [Acceso 20 nov 2018]; 34(1): 53-9. Disponible en: http://www.scielo.org.mx/scielo.php?script=sci_ arttext\&pid=S0185-33252011000100007\&lng=es.

20. Seguel F, Santander G, Alexandre O. Validez y confiabilidad del test de identificación de los trastornos debidos al consumo del alcohol (AUDIT) en estudiantes de una Universidad Chilena. Cienc Enferm. [Internet]. 2013 [Acceso 20 nov 2018]; 19(1): 23-35. Disponible en: https://scielo.conicyt.cl/scielo.php?script=sci_ arttext\&pid=S0717-95532013000100003\&Ing=es. http://dx.doi.org/10.4067/S0717-95532013000100003. 21. Alvarado ME, Garmendia ML, Acuña G, Santis R, Arteaga Validez y confiabilidad de la versión chilena del Alcohol Use Disorders Identification Test (AUDIT). Rev Méd Chilena. [Internet]. 2009 Nov [Acceso 20 no9v 2018]; 137(11): 1463-8. Disponible en: https://scielo. conicyt.cl/scielo.php?script=sci_arttext\&pid=S0034$98872009001100008 \&$ Ing=es. http://dx.doi. org/10.4067/S0034-98872009001100008.

22. Castaño-Pérez GA, Calderón-Vallejo GA. Problemas asociados al consumo de alcohol en estudiantes universitarios. Rev. Latino-Am. Enfermagem. [Internet]. 2014 Sept.-Oct [Acceso 20 nov 2018]; 22(5): 739-76. Disponible en: http://www.scielo. br/scielo.php?script=sci_arttext\&pid=S0104$11692014000500739 \& / n g=e n$. http://dx.doi. org/10.1590/0104-1169.3579.2475

23. Bautista-Álvarez TM, Pimentel-Jaimes JA, TelumbreTerrero JY, Sánchez-Jaimes BE. Consumo de alcohol en estudiantes de nivel medio superior. Rev Adicc Cienc. [Internet]. 2016 [Acceso 20 nov 2018]; 4):1-15. Disponible en: http://adiccionyciencia.info/consumo-dealcohol-en-estudiantes-de-nivel-medio-superior-alcoholconsumption-on-students-of-medium-level-superior 
24. Vargas-Valle E, D. Martínez-Canizales G. La relación entre el abuso del alcohol y la religión e los adolescentes mexicanos. Población Salud Mesoamérica. [Internet]. 2015; [Acceso 20 nov 2018]; 12(2):1-21. Disponible en: http://www.redalyc.org/articulo. oa?id $=44633024008$ doi: $10.15517 / p s m . v 12 i 2.16783$

25. Ruiz-Torres GM, Medina-Mora Icaza ME. La percepción de los adolescentes sobre el consumo de alcohol y su relación con la exposición a la oportunidad y la tentación al consumo de alcohol. Salud Mental. [Internet]. 2014 Feb [Acceso 20 nov 2018]; 37(1): 1-8. Disponible en: http://www.scielo.org.mx/scielo.php?script=sci_ arttext\&pid=S0185-33252014000100001\&lng=es. 